\title{
EFECTO DE LA ALTURA EN LA RADIACIÓN SOLAR ULTRAVIOLETA EN ARICA NORTE DE CHILE ${ }^{1}$
}

\author{
Miguel Rivas A. ${ }^{1-2} \quad$ Elisa Rojas E. $^{2} \quad$ Juan Cortés N. $^{3} \quad$ Elisa Santander G. ${ }^{3}$
}

Recibido el 16 de enero de 2002, aceptado el 12 de septiembre de 2002

\begin{abstract}
RESUMEN
En este trabajo se presentan resultados obtenidos al calcular mediante el modelo TUV (Tropospherical Ultraviolet Visible Model) las dosis eritémicas y los índices de radiación solar ultravioleta que se reciben a nivel del mar en Arica (Lat.: $18^{\circ}$ 49’ S; Long. $70^{\circ} 19^{\prime}$ W) y zonas del altiplano. Entre los resultados más destacados obtenidos podemos mencionar: un aumento promedio en las dosis eritémicas de un $5.6 \% / \mathrm{km}$, lo cual muestra un buen acuerdo con datos experimentales, en los cálculos correspondientes a los índices de radiación solar ultravioleta encontramos que en ausencia de nubosidad el modelo teórico predice valores superiores a 20 ( en una escala dónde valores de índice ultravioleta superiores a 14 son considerados extremos). Estos valores calculados por el modelo se observan sobre los $1900 \mathrm{~m}$ de altitud y durante gran parte del año (para días de cielo claro). El tema es de especial interés debido al creciente número de personas que se desplazan continuamente entre el nivel del mar y altitudes cercanas a los $5000 \mathrm{~m}$ de altura, esto se debe a trabajos relacionados con proyectos de la gran minería del cobre, como también a labores relativas al turismo. En ambos casos estas personas reciben importantes incrementos en los niveles de radiación solar ultravioleta.
\end{abstract}

Palabras Claves: Ozono, radiación, ultravioleta

\begin{abstract}
In this work, using transfer radiative models, we present results obtained when calculating the levels of solar ultraviolet radiation that reach Arica at sea level, as well as altitudes of $4.500 \mathrm{~m}$ above sea level. (Lat.: 18 49' S; Long. $70^{\circ} 19^{\prime} \mathrm{W}$ ). The level of solar ultraviolet radiation that reaches the earth varies due to multiple factors, such as the geographical location (latitude), the thickness of the ozone layer present above, the cloud cover and the altitude above sea level. Locations at both sea level and at $4500 \mathrm{~m}$ above sea level can be found in northern Chile. These locations are specially adequate for the study of the effects of altitude in the increase of solar ultraviolet radiation. This topic is relevant because a great number of people, having jobs related to copper mining and tourism, who often travel moving from places that are located at sea level to settings that are situated at $5000 \mathrm{~m}$ above sea level, are exposed to significantly increased solar ultraviolet radiation.
\end{abstract}

Keywords: Ozone, radiation, ultraviolet

\section{INTRODUCCIÓN}

El deterioro progresivo que ha sufrido la capa de ozono en las últimas dos décadas Herman [1], [2], Bojkov [3], 1995) puede tener consecuencias importantes en los seres vivos en la Tierra, ante un incremento eventual de la radiación solar ultravioleta B (UVB: 280-320 nm atenuada por la capa de ozono Herman [4], [5]. Estas radiaciones tienen bastante energía para producir daño y perjuicios en sistemas bológicos. Bajo condiciones normales, radiaciones de longitudes de onda menores que $290 \mathrm{~nm}$ no llegan prácticamente a la superficie de tierra. Cada especie de ser vivo, incluyendo individuos diferentes dentro de una misma especie, tienen una respuesta diferente a una irradiancia solar ultravioleta (UV) dada Tevini [6]. A su vez, partes diferentes del mismo organismo responden de una manera específica, de tal manera que el estudio de la exposición natural a la

\footnotetext{
${ }^{1}$ Centro de Investigaciones del Hombre en el Desierto, Universidad de Tarapacá, Avenida General Velásquez 1775, Arica, Chile.

${ }^{2}$ Universidad de Tarapacá, Departamento de Física, Casilla 7-D, mrivas@uta.cl, erojas@utacl, Fax (58) 205435, Arica, Chile.

${ }^{3}$ Universidad de Tarapacá, Departamento de Química, Casilla 7-D, jcortes@uta.cl, esantander@uta.cl.
} 
radiación solar ultravioleta en este rango de longitudes de onda constituye un campo entero en sí mismo.

La vecindad del extremo Sur de nuestro Hemisferio con la región Antártica, donde anualmente tiene lugar el evento denominado "agujero en la capa de ozono" en la época invierno-primavera, donde se destruye el porcentaje más grande de la capa de ozono del planeta Eck [7], hace esencial el estudiar la evolución de las variables directamente relacionadas con este fenómeno, en la zona Norte de nuestro país lugar geográfico correspondiente a latitudes medias y altas. En el caso del ozono estratosférico, la medición con instrumentos ubicados en satélites ha alcanzado gran precisión y ha permitido un muestreo permanente del planeta entero. Es importante destacar que de estas mediciones se han derivado niveles de irradiación solar UV y de dosis eritémicas en toda la Tierra a partir de los datos satelitales del instrumento TOMS/NASA Herman [8]. Es importante considerar que estas cantidades son mucho más sensibles al estado de la atmósfera y dependen de múltiples factores tales como: nubes, aerosoles, reflectividad del suelo (albedo), altura del lugar, espesor de la capa de ozono, etc., todos los cuales hacen mucho más complejo su estudio. El modelamiento teórico también es una herramienta de análisis importante y permite contrastar con datos de estaciones de tierra y satelitales. Por consiguiente, tanto para la contrastación de datos satélites como para la comprobación de modelos, es de gran importancia tener instrumentos y mediciones lo más preciso posible. Hay dos tipos de instrumentos físicos para medir la irradianza solar ultravioleta (UV): los instrumentos de banda angosta con muy buena resolución espectral que permiten cubrir una región del espectro con una separación de espectro fija en cuanto a longitudes de onda (ellos miden en longitudes de onda fijas con muy buena resolución espectral). En nuestro país existen datos experimentales tomados con este tipo de instrumentos en Santiago por Cabrera [9]. Los instrumentos de banda ancha que miden simultáneamente un ancho de banda amplio de longitudes de onda con una respuesta espectral cercana a la función respuesta de algún sistema biológico (normalmente se llaman biómetros. En este trabajo presentamos los resultados obtenidos por medio de modelos en lugares que van entre el nivel del mar y los $7000 \mathrm{~m}$ de altura. Las simulaciones se realizaron mediante un modelo matemático paramétrico que permite obtener las variaciones relativas y las diferencias porcentuales entre las dosis eritémicas que se obtuvieron en los diferentes lugares en función de la altitud del lugar.

\section{MODELO MATEMÁTICO}

El modelo matemático utilizado en este trabajo corresponde al modelo TUV (Tropospherical Ultraviolet Visible Model. El modelo TUV fue desarrollado por Madronich [10] en la División de Química Atmosférica del Centro Nacional para la Investigación de la Atmósfera en USA.

El modelo TUV permite realizar cálculos en el rango de longitudes de onda de 121 - $750 \mathrm{~nm}$ para el cálculo de: irradianzas espectrales, flujo espectral, coeficientes de fotodisociación y la irradianza efectiva biológicamente (dosis eritémica).

El modelo TUV, permite además el cálculo de un importante número de variables entre los cuales podemos destacar. Contribuciones directas y difusa, posibilidades de realizar simulaciones en las cuales se pueden variar las longitudes de onda, la altitud. Evaluar la dependencia de la temperatura y la presión, absorción de ozono y de dióxido de sulfuro, el efecto de scattering debido al cambio de densidad del aire que compone. El modelo TUV permite incorporar, si se cuenta con información meteorológica el efecto de la nubosidad sobre las dosis eritémicas que se miden a nivel del suelo sobre un determinado lugar geográfico, además el modelo considera el efecto de los aerosoles (contribuciones de Rayleigh y de Mie) sobre la radiación solar ultravioleta que se recibe a nivel del suelo. El código en el cual está escrito el programa es Fortran-77.

El modelo trabaja con el perfil de temperaturas del aire estándar de Estados Unidos obtenido en 1976. En caso de contar con perfiles de similar rango de validez, más recientes es posible incorporarlos en el programa con la debida autorización del autor (Madronich, NCAR, USA).

El modelo TUV considera la curvatura de la tierra, variable importante cuando el Sol se encuentra cerca del atardecer o del amanecer.

Finalmente podemos indicar que según el interés de quienes utilizan el programa el modelo TUV calcula otras cantidades derivadas tales como el factor de amplificación de radiación (RAF).

La columna total de ozono tiene una dependencia con la latitud que el modelo considera y se consideran valores promedios calculados para cada lugar geográfico de la tierra a partir de las tendencias de las últimas décadas obtenidas mediante estudios de estaciones de tierra y datos satelitales.

Tabla 1.-Índice de riesgo solar UV para diferentes altitudes de la Región del desierto de Atacama $18.5^{\circ} \mathrm{S}$ 


\begin{tabular}{c|c|c|c|c|c|c|}
\hline $\begin{array}{c}\text { Altura } \\
\text { (Km.) }\end{array}$ & 0 & 1100 & 1500 & 1900 & 3400 & 4400 \\
\hline Enero & 10,8 & 16,4 & 17,2 & 18,0 & 20,8 & 22,6 \\
\hline Feb. & 10,4 & 15,4 & 6,5 & 17,0 & 19,6 & 21,6 \\
\hline Marzo & 8,4 & 13,0 & 5,4 & 14,2 & 16,8 & 18,4 \\
\hline Abril & 6,2 & 10,0 & 4,2 & 11,0 & 13,2 & 14,6 \\
\hline Mayo & 4,6 & 7,8 & 3,3 & 8,6 & 10,6 & 11,8 \\
\hline Junio & 4,4 & 7,4 & 3,1 & 8,2 & 10,0 & 11,2 \\
\hline Julio & 5,4 & 8,9 & 3,7 & 9,6 & 11,6 & 13,0 \\
\hline Agos. & 7,4 & 11,6 & 4,8 & 12,4 & 15,2 & 16,6 \\
\hline Sept. & 9,4 & 14,4 & 6,0 & 15,6 & 18,2 & 20,0 \\
Oct. & 10,6 & 16,0 & 6,7 & 17,6 & 20,2 & 22,0 \\
\hline Nov. & 10,8 & 16,4 & 6,9 & 17,8 & 20,8 & 22,4 \\
\hline Dic. & 7,2 & 16,4 & 6,8 & 18,0 & 20,8 & 22,4 \\
\hline
\end{tabular}

El modelo TUV es de muy fácil manejo y permite ingresar al programa otras variables que tienen influencia en la irradiancia solar ultravioleta. Por ejemplo: contenidos totales de aerosoles de la atmósfera, el albedo (reflectividad de la superficie del lugar.

\section{RESULTADOS OBTENIDOS}

La Tabla 1. Muestra el índice de riesgo Solar UV para diferentes altitudes de la Región de Atacama $\left(18.5^{\circ} \mathrm{S}\right)$ y diferentes meses del año correspondientes a los períodos de equinoccio y solsticio.

En la Tabla 2 se muestra la calificación de riesgo Solar para los rangos diferentes del índice UV. La calificación de riesgo solar medida a través del índice UV se encuentra multiplicando por un factor 40 la irradianza eritémica medida en $\left(\mathrm{W} / \mathrm{m}^{2}\right)$.

Tabla 2: Calificación de riesgo solar para rangos diferentes del Índice UV

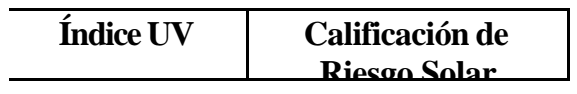

\begin{tabular}{|c|c|}
\hline $0-2.9$ & Muy Bajo \\
$3-4.9$ & Bajo \\
\hline $5-6.9$ & Moderado \\
\hline $7-9.9$ & Alto \\
\hline $10-13.9$ & Muy Alto \\
\hline 14 or more & Extremo \\
\hline
\end{tabular}

En la Fig. 1 se muestran las dosis eritémicas calculadas mediante el modelo de transferencia radiativa en función de la altitud sobre el nivel del mar para las distintas estaciones del año.

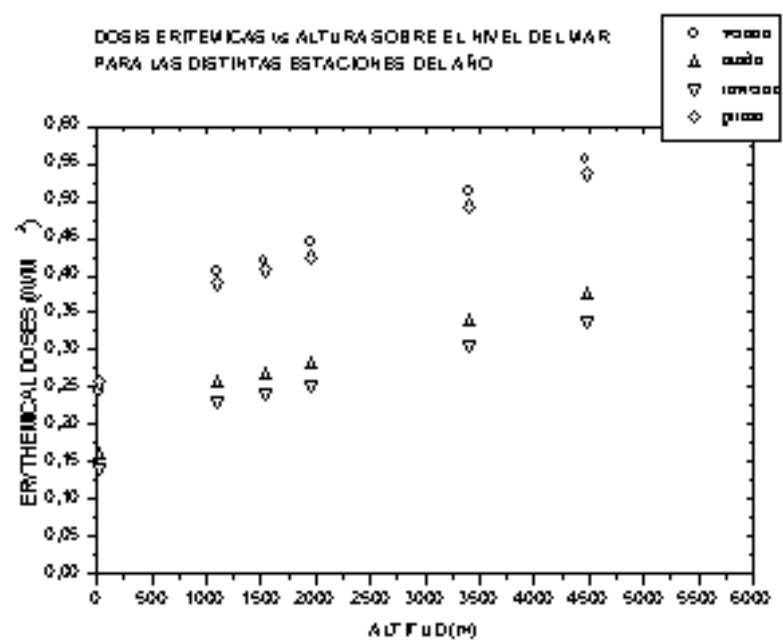

Fig. 1.- Dosis eritémicas calculadas mediante el modelo de transferencia radiativa

\section{CONCLUSIONES}

Una curva promedio derivada de los resultados obtenidos al calcular durante todos los meses del año nos da un aumento promedio de dosis eritémica de un $5.6 \% / \mathrm{Km}$. para cálculos realizados sobre superficies con ausencia de nieve $y$ en condiciones normales correspondientes a días despejados en ausencia de nubosidad. Los datos experimentales obtenidos en Arica $23 \mathrm{~m}$ y Zapahuira $3170 \mathrm{~m}$ por Rivas [11], se muestran en la Tabla 3 en la cual se muestran los incrementos en las dosis eritémicas obtenidos por $\mathrm{Km}$.

Tabla 3.- Datos experimentales de Dosis Eritémicas medidas en Arica y Zapahuira 


\begin{tabular}{|c|c|c|c|c|}
\hline $\begin{array}{c}\text { Local } \\
\text { Time }\end{array}$ & $\begin{array}{c}\text { Arica } \\
\mathrm{I}\left(\mu \mathrm{w} / \mathrm{cm}^{2}\right)\end{array}$ & $\begin{array}{c}\text { Zapahuira } \\
\left(\left(\mu \mathrm{w} / \mathrm{cm}^{2}\right)\right.\end{array}$ & $\begin{array}{c}\% \\
\text { Difference }\end{array}$ & $\begin{array}{c}\% \\
\text { Difference/km }\end{array}$ \\
\hline $10: 58$ & 5.91 & 7.85 & 32.8 & 14.9 \\
\hline $11: 30$ & 7.43 & 8.82 & 18.7 & 8.5 \\
\hline $11: 45$ & 7.49 & 9.15 & 22.2 & 10.1 \\
\hline $12: 00$ & 7.76 & 9.46 & 21.9 & 10.0 \\
\hline $13: 15$ & 7.59 & 9.71 & 27.9 & 12.7 \\
\hline $13: 45$ & 7.18 & 9.23 & 28.6 & 13.0 \\
\hline $14: 30$ & 5.76 & 7.93 & 37.7 & 17.1 \\
\hline & & & $\begin{array}{c}\text { Mean } \\
\text { Value }\end{array}$ & 12.3 \\
& & & \multicolumn{3}{|l}{} \\
\hline
\end{tabular}

[3] R. D. Bojkov, L. Bish op and V.E. Fioletov; “Total Ozone Trends from Quality-Controlled Ground Base Data (1964-1994)", J. Geophys. Res.,Vol. 100, pp. 25867-25876, 1995.

[4] J. R. Herman, P.K. Barthia, J. Ziemke, Z. Ahmad and D. Larko; "UV-B Increases (1979-1992) from Decreases in Total Ozone", Geophys. Res. Lett., Vol. 23, pp 2117, 1996.

[5] J. R. Herman and E. Celarier; TOMS Version 7; "UVErythemal exposure": 1978-1993, CD edited by NASA, Goddard Space Flight Center, 1996.

La comparación realizada con los cálculos obtenidos mediante el modelo TUV para días de cielo claro, muestra un buen acuerdo con los datos experimentales. Una consecuencia importante de nuestros resultados es la obtención de valores muy altos de dosis eritémicas en el altiplano durante las estaciones del año correspondientes a primavera y verano para días de cielo claro y en horas cercanas al mediodía solar. Estos altos valores de dosis eritémicas fueron obtenidos por el modelo en esta región para todas las altitudes, el modelo de transferencia radiativa indica valores de índice solar ultravioleta cercanos a 20 para zonas del altiplano, estos índices corresponden a valores extremos. De acuerdo a estos resultados de valores extremos del índice ultravioleta en zonas del altiplano, creemos importante a futuro poder realizar comparaciones entre el modelo TUV $\mathrm{y}$ datos experimentales obtenidos en otras latitudes.

De acuerdo a nuestros resultados teóricos consideramos importante recomendar mantener medidas de protección a la exposición de la piel y la vista a la radiación solar ultravioleta B (UVB) para las personas que viajan al altiplano de nuestra región aún en los meses de invierno, ya que de acuerdo al modelo, aún durante estos meses se obtienen valores del índice solar ultravioleta considerados altos o extremos.

\section{REFERENCIAS}

[1] J. R. Herman, R. Hudson, R. McPeters, R. Stolarski, Z. Ahmad, X. Y. Gu, S. Taylor and C. A. Wellemeyer,; "New Self-Calibration Method Applied to TOMS and SBUV Backscattered Ultraviolet Data to Determine Long-Term Global Ozone Change", J. Geophys. Res., Vol. 96, pp 7531-7545, 1991.

[2] J. R. Herman, R. McPeters and D. Larko; "Ozone Depletion at Northern and Southern Latitudes Derived from January 1979 to November 1991, Total Ozone Mapping Spectrometer", J. Geophys. Res., Vol. 98, pp. 12783, 1993. Effects on Humans, Animals, Plants, Microorganism and Materials", Lewis Praeguer Publishers, 1993.

[7] T. F. Eck, P.K. Bhartia and J.B. Kerr; "Satellite Estimation of Spectral UVB irradiance Using TOMS Derived Total Ozone and UV Reflectivity", Geo. Res. Lett. Vol. 22, pp 611-614, 1995.

[8] J. R. Herman, P.K. Barthia, Z. Ahmad and D. Larko; "Change in 300 to $340 \mathrm{~nm}$. Solar Ultraviolet Exposure at the Ground Between (1980-1993) Estimated from NIMBUS7/TOMS Ozone Data”, Private communication, 1994.

[9] S. Cabrera, H. Fuenzalida; "Interannual Variations of Global UV Radiation in Santiago Chile (33.5 S)", Geophys. Res. Lett., Vol. 26, pp. 2945-2948, 1999.

[10] S. Madronic, R. Mckenzie, J. Bjorn, and M. Caldwell; "Changes in Ultraviolet Radiation Reaching the Earth's surface”, Ambio Vol. 24, pp. 143-158, 1995.

[11] M. Rivas, E. Rojas, R. Piacentini and J. Herman; "Dosis Eritémicas Solares Medidas en el Norte de Chile en el Solsticio de Verano de 1995", Actas de Asades, 1995. 